(2) Open Access Full Text Article

CLINICALTRIAL REPORT

\title{
Peripheral Blood Eosinophil as a Biomarker in Outcomes of Acute Exacerbation of Chronic Obstructive Pulmonary Disease
}

This article was published in the following Dove Press journal: International Journal of Chronic Obstructive Pulmonary Disease

\author{
Hong-Xia $\mathrm{Wu}^{\mathrm{I}, *}$ \\ Kai-Quan Zhuo ${ }^{2, *}$ \\ De-Yun Cheng' ${ }^{1} *$
}

'Department of Respiratory and Critical Care Medicine, West China Hospital, Sichuan University, Chengdu, People's Republic of China; ${ }^{2}$ Department of Neurosurgery, Suining Municipal Hospital of TCM, Suining, People's Republic of China

*These authors contributed equally to this work
Correspondence: De-Yun Cheng Department of Respiratory and Critical Care Medicine, West China Hospital, Sichuan University, No. 37 Guoxue Alley, Chengdu 61004I, Sichuan, People's Republic of China

Email chengdeyunscu@I63.com
Purpose: Mounting evidence suggests that eosinophil levels correlate with the effects of therapy and phenotype for chronic obstructive pulmonary disease (COPD). This study aimed to clarify the relationship between eosinophil levels and clinical outcomes in patients with acute exacerbation of COPD (AECOPD).

Methods: A prospective, multicenter, observational cohort study was performed in three teaching hospitals. Patients were grouped by quartile percentage $(0,0.7,2.55)$ and absolute blood eosinophils count $\left(0,0.05 \times 10^{9} / \mathrm{L}, 0.17 \times 10^{9} / \mathrm{L}\right)$ and divided into four numbered groups ranked from low to high.

Results: The study included 493 AECOPD patients. In the percentile-ranked groups, patients in Group 1 experienced significantly longer hospital stays, higher rates of both noninvasive mechanical ventilation (NIMV), and heart failure than those in Group 4 (12 days vs 10 days, $\mathrm{p}=0.005 ; 29.5 \%$ vs $23.6 \%, \mathrm{p}=0.007 ; 48.4 \%$ vs $28.5 \%, \mathrm{p}=0.001$ ). Group 1 also had higher frequencies of respiratory failure and pulmonary heart disease compared to Groups 3 and $4(54.8 \%$ vs $34.8 \%, p=0.002 ; 54.8 \%$ vs $35 \%, p=0.003)$. In the absolute count-ranked groups, patients in Group 1 had significantly higher rates of NIMV than those in Group 3 (41.1\% vs $21.7 \%, \mathrm{p}=0.001)$, had higher rates of heart failure, respiratory failure, and pulmonary heart disease than those in Group 3 and $4(48.1 \%$ vs $30.2 \%, p=0.003 ; 48.1 \%$ vs $30.4 \%, \mathrm{p}=0.005 ; 50.8 \%$ vs $32.2 \%, \mathrm{p}=0.004 ; 50.8 \%$ vs $34.1 \%, \mathrm{p}=0.008 ; 51.9 \%$ vs $34.1 \%, \mathrm{p}=0.004 ; 51.9 \%$ vs $33 \%, \mathrm{p}=0.003)$. There were outcome differences among the admitting hospital of stays in the absolute count groups $(\mathrm{p}=0.002)$, but the differences were not significant in a pairwise comparison. The proportion of ICU admissions and mortality was different in two cohorts with no difference in a pairwise comparison.

Conclusion: Patients with lower eosinophil counts experienced poorer clinical outcomes. Eosinophil levels may be a helpful marker to predict outcomes in AECOPD.

Keywords: eosinophils, chronic obstructive pulmonary disease, exacerbation, biomarkers, mortality

\section{Introduction}

Chronic obstructive pulmonary disease (COPD) is a fatal disease that is projected to be the third most common cause of death worldwide within the next three years. ${ }^{1}$ COPD is a heterogeneous disease that exhibits complex pathological features. Exacerbations of COPD are defined as an acute worsening of respiratory symptoms resulting in a need for additional therapy. ${ }^{2,3}$ Aside from neutrophilic inflammation, eosinophilic inflammation is a new area of research. The latest studies demonstrated that eosinophilic inflammation exists in both stable and acute exacerbations of 
COPD (AECOPD) ${ }^{4,5}$ Mounting evidence suggests that eosinophil levels may be related to the effects of therapy and outcomes, even in the absence of asthma. ${ }^{6-9}$ Blood eosinophils are usually used as a biomarker for response to inhaled steroids (ICS) and exacerbation risk in stable COPD. ${ }^{10-16}$ Bafadhel et al found that eosinophil count $(100 / \mu \mathrm{L}$ and $300 / \mu \mathrm{L})$ predicted the risk of exacerbations and the response to treatment with ICS in patients with COPD. ${ }^{17}$ Blood eosinophil counts are recommended by the Global Initiative for Chronic Obstructive Lung Disease (GOLD) as a biomarker to guide ICS therapy in clinical practice. ${ }^{1}$ However, the effect of blood eosinophil in stable and acute cases may be different.

Saltürk et al reported that patients with eosinophil levels less than $2 \%$ experienced shorter intensive care unit (ICU) stays and lower mortality rates. ${ }^{18}$ Similarly, Kang suggested better pulmonary function, lower admissions to the ICU, and mortality in a group with eosinophil levels less than $2 \% .{ }^{19}$ Duman et al reported that shorter hospital stays and lower readmission rates were found in the group with eosinophilia, but no differences were found in six-month mortality. ${ }^{20}$ Patients with AECOPD, and low $(<50 / \mu \mathrm{L})$ eosinophil were strongly associated with longer median hospital stay ( 7 vs 4 days, $\mathrm{P}<0.001$ ), and lower 12 -month survival $(82.4 \%$ vs $90.7 \%, \mathrm{P}<0.028)$ than patients with high $(>150 / \mu \mathrm{L})$ eosinophil counts. ${ }^{21}$ An eosinophil value of $<0.144 \times 10^{9} / \mathrm{L}$ (or less than $2 \%$ ) on admission was associated with a longer hospital stay for AECOPD. ${ }^{22}$

However, the effect of blood eosinophil in AECOPD outcomes remains controversial. Two studies found longer hospitalization, ${ }^{23,24}$ a greater need for mechanical ventilation, ${ }^{23}$ and increased mortality ${ }^{23,24}$ in the group with eosinophilia. Further, a higher frequency of readmission for $\mathrm{AECOPD}^{25}$ and a higher rate of exacerbations ${ }^{26}$ have been found in those with eosinophilia. This study aimed to investigate the effect of peripheral blood eosinophil in patients who experienced AECOPD.

\section{Methods}

This prospective, multicenter study was conducted in three university-affiliated hospitals in China. Patients who were hospitalized for AECOPD between September 2018 and February 2019 were enrolled. The study was approved by the local ethics committee (Biomedical Ethics Committee of West China Hospital of Sichuan University) and complied with the Declaration of Helsinki. All patients provided written informed consent. The study was registered in the
Chinese Clinical Trial Registry (ChiCTR1900024210). All data were collected from questionnaire surveys and hospital databases.

\section{Subject}

The definitions of COPD were based on the GOLD criteria. Spirometry is required to diagnose COPD. The presence of a post-bronchodilator $\mathrm{FEV}_{1} / \mathrm{FVC}$ ratio $<0.70$ confirms the presence of persistent airflow limitation and, thus, COPD in patients with appropriate symptoms and significant exposures to noxious stimuli. ${ }^{1}$ COPD exacerbations are defined as an acute worsening of respiratory symptoms that result in additional therapy. ${ }^{1}$ Inclusion criteria were as follows: $\geq 40$ years of age with AECOPD; routine baseline peripheral blood test was performed before receiving any antibiotic or systemic corticosteroid therapy (prednisone $>0.5 \mathrm{mg} / \mathrm{kg}$ or equivalent doses). Patients admitted due to other medical problems, those with a history of asthma, active pulmonary tuberculosis, interstitial pulmonary disease or lung cancer, those undergoing chronic oral steroid therapy, those with other diseases that could influence eosinophil count (allergic diseases, parasitic infections, eosinophilic pneumonia), and individuals with severe dysfunction of other organs or systems or malignant tumors were excluded.

\section{Measurements}

Patient baseline characteristics, including age, sex, body mass index, allergy history, smoking history, duration of disease, long-term home oxygen therapy, regular medications, heart and respiratory rate on admission, comorbidities (hypertension, diabetes mellitus, arrhythmia, chronic ischemic heart disease, congestive heart failure, peripheral vascular disease, bronchiectasis, respiratory failure, and pulmonary heart disease), manner of hospital admission, the number of hospital or emergency admissions in the previous year, laboratory data (routine blood test and arterial blood gas analysis), admission to the ICU, length of hospital stay, rate, and duration of noninvasive mechanical ventilation (NIMV), and hospital medical treatment and mortality, were recorded. A COPD assessment test (CAT), modified British Medical Research Council (mMRC), and the refined ABCD assessment were also evaluated using questionnaires. The primary outcome measure was the length of the hospital stay. Secondary outcome measures included ICU admission rate, and duration of noninvasive ventilation, comorbidities, and mortality. 


\section{Analysis}

Clinical outcomes were compared among patients grouped according to quartile-percent and absolute count of peripheral blood eosinophils (From low to high: Groups 1, 2, 3, 4). Pearson's chi-squared test or Fisher's exact was used to compare discrete variables. The Kruskal-Wallis test was used for pairwise comparisons if differences were revealed using the chi-squared test. Analysis of variance and nonparametric tests were used to compare continuous variables. Kaplan-Meier analysis was performed to identify the associated factors and hospital length of stay. The receiver operating characteristic (ROC) curve with the calculation of the area under the curve (AUC) was used to identify the cutoff values of eosinophils associated with longer hospital lengths of stay. A two-sided $\mathrm{P} \leq 0.05$ was statistically significant. One-sixth of the P-value $\leq 0.05$ was statistically significant in the Kruskal-Wallis test. All statistical analyses were performed using SPSS version 25.0 (IBM Corporation, Armonk, NY, USA).

\section{Results}

Overall, 1099 COPD patients who experienced acute exacerbations were admitted during the study period, of whom 493 were analyzed (Figure 1). Medians of percentage and absolute count of peripheral blood eosinophils were $0.7 \%$ (interquartile range [IQR] $0-2.55$ ) and $0.05 \times 10^{9} / \mathrm{L}$ (IQR $0-0.17)$ in total. Patients were classified according to percentage of eosinophil quartile as follows: Group $1(n=124)$, Group $2(\mathrm{n}=131)$, Group $3(\mathrm{n}=115)$, and Group 4 $(\mathrm{n}=123)$. Similarly, patients were classified according to eosinophil count quartile as follows: Group $1(\mathrm{n}=129)$, Group $2(\mathrm{n}=120)$, Group $3(\mathrm{n}=129)$ and Group $4(\mathrm{n}=$ 115). The proportion of males in the present study was $69.2 \%$. The median (IQR) age, BMI, course of disease, and length of hospital stay were 76 (68-83) years, 21.224 (18.5-24.315) kg/m², 10 (5-20) days and 11(9-14) days, respectively. The clinical characteristics and laboratory findings on admission of the patients are summarized in Tables 1-4. AECOPD was treated with oxygen therapy, atomization, antibiotics, or systemic steroids. Antibiotics and systemic steroids were prescribed at the discretion of the attending physician.

The primary outcome measure was the length of the hospital stay. The length of hospital stays was found to be different in the two types of groups $(p=0.01, p=0.002)$ (Tables 5 and 6). In pairwise comparison, a significantly longer hospital stay was found in Group 1 than in Group 4

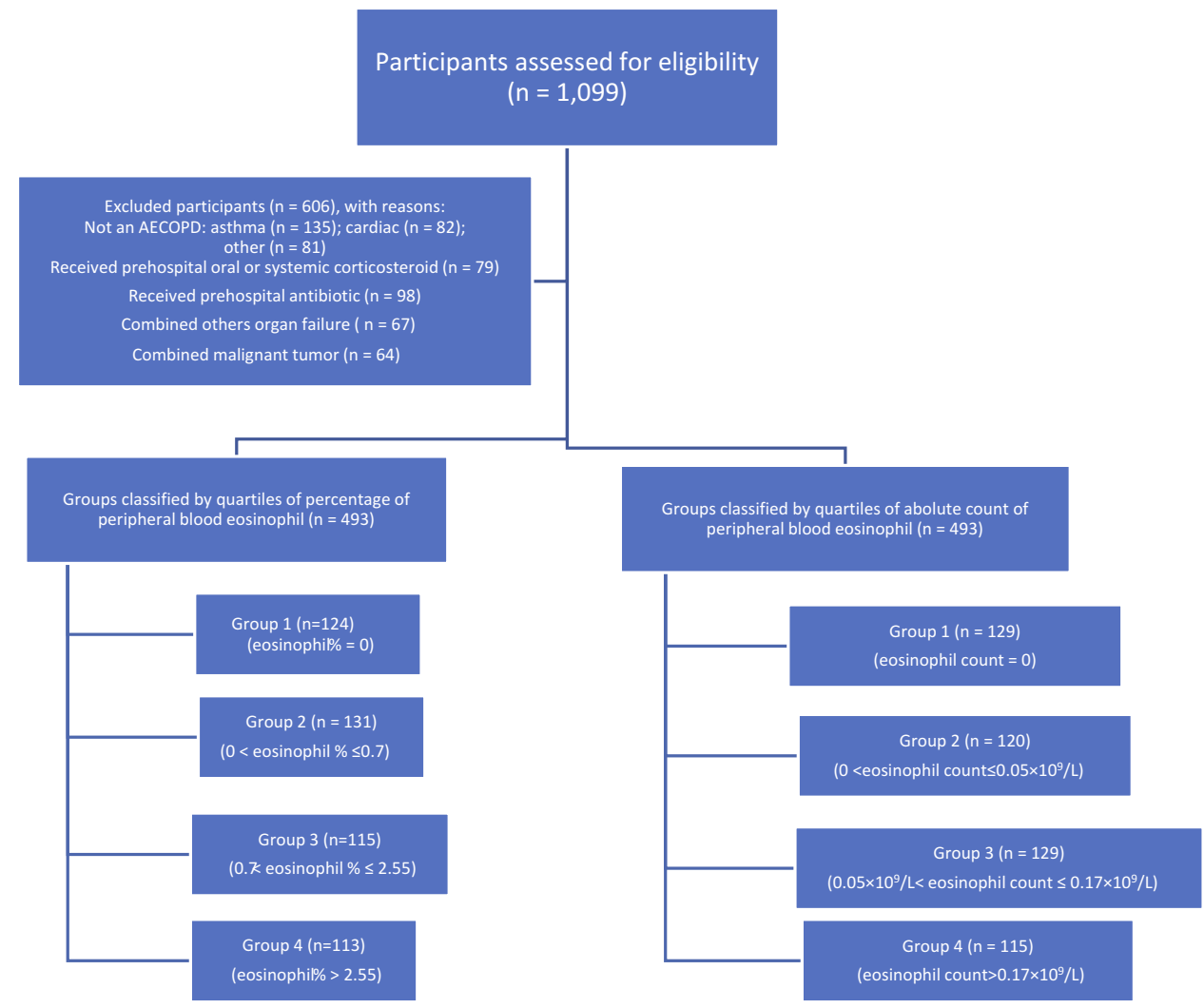

Figure I Flow chart of subjects. 
Table I Patients' Characteristics on Admission of Quartile-Percentage of Eosinophil Cohorts

\begin{tabular}{|c|c|c|c|c|c|c|}
\hline \multirow{2}{*}{$\frac{\text { Variables }}{\text { Groups }}$} & & \multicolumn{5}{|c|}{ Percentage of Peripheral Blood Eosinophil ${ }^{a}$} \\
\hline & & Overall & $\mathbf{I}$ & 2 & 3 & 4 \\
\hline Participants, $\mathrm{n}$ & & 493 & 124 & $13 \mid$ & 115 & 123 \\
\hline \multirow[t]{2}{*}{ Gender, n (\%) } & Female & $149(30.2)$ & $38(30.6)$ & $39(29.8)$ & $37(32.2)$ & $35(28.5)$ \\
\hline & Male & $344(69.8)$ & $86(69.4)$ & $92(70.2)$ & $78(67.8)$ & $88(71.5)$ \\
\hline Year, median (IQR) & & $76(68-83)$ & $76(69-83)$ & $75(66-81)$ & $77(67-83)$ & $77(69-82)$ \\
\hline - BMI, median (IQR) & & $\begin{array}{l}21.224 \\
(18.5-24.315)\end{array}$ & $\begin{array}{l}21.1 \\
(17.8-24.22)\end{array}$ & $\begin{array}{l}21.09 \\
(18.75-23.88)\end{array}$ & $\begin{array}{l}20.98 \\
(18.83-24.86)\end{array}$ & $\begin{array}{l}21.71 \\
(18.59-24.73)\end{array}$ \\
\hline - Course of disease, year, median (IQR) & & $10(5-20)$ & $10(5-29)$ & $10(5-20)$ & $10(5-15)$ & $10(5-10)$ \\
\hline - Allergic history, n (\%) & & $15(3)$ & $3(2.4)$ & $9(6.9)$ & $\mathrm{I}(0.9)$ & $2(1.6)$ \\
\hline \multirow[t]{4}{*}{ - Smoking history, n (\%) } & Current smoking & $40(8.1)$ & $15(12.1)$ & $\mathrm{II}(8.4)$ & $8(7)$ & $6(4.9)$ \\
\hline & Ex-smoking & $273(55.4)$ & $67(54)$ & $69(52.7)$ & $68(59.1)$ & $69(56.1)$ \\
\hline & No-smoking & $180(36.5)$ & $42(33.9)$ & $5 I(38.9)$ & $39(33.9)$ & $48(39)$ \\
\hline & $\begin{array}{l}\text { Smoking index, } \\
\text { median }\end{array}$ & $\begin{array}{l}600 \\
(400-900)\end{array}$ & $600(300-800)$ & $\begin{array}{l}600 \\
(400-1000)\end{array}$ & $\begin{array}{l}600 \\
(300-1000)\end{array}$ & $\begin{array}{l}600 \\
(300-900)\end{array}$ \\
\hline HR, median (IQR) & & $88(78-96.5)$ & $90(80-98.75)$ & $89(80-96)$ & $86(77-97)$ & $82(76-93)$ \\
\hline RR, median (IQR) & & $20(20-21)$ & $20(20-22)$ & $20(20-21)$ & $20(20-2 I)$ & $20(20-21)$ \\
\hline LTOT, n (\%) & & $246(49.9)$ & $70(56.5)$ & $7 \mid(54.2)$ & $53(46.1)$ & $52(42.3)$ \\
\hline \multirow[t]{3}{*}{ - Daily treatment } & ICS, n (\%) & $176(35.7)$ & $48(38.7)$ & $50(38.2)$ & $36(31.3)$ & $42(34.1)$ \\
\hline & LABA, n (\%) & 176(35.7) & $48(38.7)$ & $50(38.2)$ & $36(31.3)$ & $42(34.1)$ \\
\hline & LAMA, n (\%) & $106(21.5)$ & $32(25.8)$ & $24(18.3)$ & $21(18.3)$ & $29(23.6)$ \\
\hline CAT, median (IQR) & & $18(14-26)$ & $19.5(\mid 4.25-27)$ & $18(14-26)$ & $19(14-26)$ & $18(13-26)$ \\
\hline mMRC, median (IQR) & & $2(I-3)$ & $2(1-3)$ & $2(I-3)$ & $2(1-3)$ & $2(I-3)$ \\
\hline $\begin{array}{l}\text { Moderate or severe exacerbation his- } \\
\text { tory in previous year, median (IQR) }\end{array}$ & & $I(0-2)$ & $\mathrm{I}(0-2)$ & $2(I-3)$ & $2(0-3)$ & $I(0-2)$ \\
\hline $\begin{array}{l}\text { - Exacerbations leading to hospital or } \\
\text { emergency admission in } \\
\text { previous year, median (IQR) }\end{array}$ & & $I(0-2)$ & $I(0-2)$ & $I(0-3)$ & $I(0-3)$ & $I(0-2)$ \\
\hline \multirow[t]{4}{*}{ ABCD assessment, $\mathrm{n}(\%)$} & A & $124(25.2)$ & $\mathrm{I}(0.8)$ & $39(31.5)$ & $2(1.6)$ & $82(66.1)$ \\
\hline & B & $|3|(26.6)$ & $2(1.5)$ & $29(22.1)$ & $2(1.5)$ & $98(74.8)$ \\
\hline & C & $115(23.3)$ & $2(1.7)$ & $34(29.6)$ & $\mathrm{I}(0.9)$ & $78(67.8)$ \\
\hline & $\mathrm{D}$ & $123(24.9)$ & $2(1.6)$ & $36(29.3)$ & $\mathrm{I}(0.8)$ & $84(68.3)$ \\
\hline \multirow[t]{2}{*}{ Pattern of admission, n (\%) } & $\begin{array}{l}\text { - Outpatient } \\
\text { service }\end{array}$ & $286(58)$ & $69(55.6)$ & $58(44.3)$ & $78(67.8)$ & $8 I(65.9)$ \\
\hline & Emergency & $207(42)$ & $55(44.4)$ & $73(55.7)$ & $37(32.2)$ & $42(34.1)$ \\
\hline
\end{tabular}

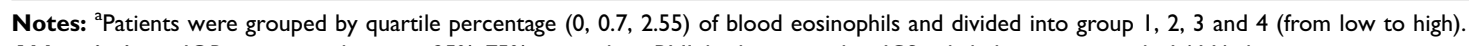

Abbreviations: IQR, interquartile range: $25 \%-75 \%$; n, number; BMI, body mass index; ICS, inhaled corticosteroids; LAMA, long-acting muscarinic antagonist; LABA, longacting beta agonist; HR, heart rate; RR, respiratory rate; LTOT, long-term oxygen therapy; CAT, COPD assessment test; mMRC, modified British medical research council. 
Table 2 Patients' Characteristics on Admission of Quartile-Count of Eosinophil Cohorts

\begin{tabular}{|c|c|c|c|c|c|c|}
\hline \multirow{2}{*}{$\begin{array}{l}\text { Variables } \\
\text { Groups }\end{array}$} & & \multicolumn{5}{|c|}{ Absolute Count of Peripheral Blood Eosinophil } \\
\hline & & Overall & $\mathbf{I}$ & 2 & 3 & 4 \\
\hline Participants, $\mathrm{n}$ & & 493 & 129 & 120 & 129 & 115 \\
\hline \multirow[t]{2}{*}{ Gender, n (\%) } & Female & $149(30.2)$ & $4 I(3 I .8)$ & $33(27.5)$ & $44(34.1)$ & $3 I(27)$ \\
\hline & Male & $344(69.8)$ & $88(68.2)$ & $87(72.5)$ & $85(65.9)$ & $84(73)$ \\
\hline Year,median (IQR) & & $76(68-83)$ & $76(69-83)$ & $74.62(9.879)^{*}$ & $74.26(9.494)^{*}$ & $77(68-83)$ \\
\hline BMI, median (IQR) & & $\begin{array}{l}21.224 \\
(18.5-24.315)\end{array}$ & $\begin{array}{l}20.96 \\
(17.78-22.22)\end{array}$ & $\begin{array}{l}20.92 \\
(18.82-23.49)\end{array}$ & $\begin{array}{l}21.41 \\
(18.79-24.92)\end{array}$ & $\begin{array}{l}21.93 \\
(21.93-24.73)\end{array}$ \\
\hline Course of disease, year, median (IQR) & & $10(5-20)$ & $10(5-20)$ & $10(5.25-20)$ & $10(3.5-10)$ & $10(5-20)$ \\
\hline Allergic history, n (\%) & & $15(3)$ & $3(2.3)$ & $7(5.8)$ & $3(2.3)$ & $2(1.7)$ \\
\hline \multirow[t]{4}{*}{ Smoking history, n (\%) } & Current smoking & $40(8.1)$ & $17(13.2)$ & $9(7.5)$ & $9(7)$ & $5(4.3)$ \\
\hline & Ex-smoking & $273(55.4)$ & $70(54.3)$ & $64(53.3)$ & $7 I(55)$ & $68(59.1)$ \\
\hline & No-smoking & $180(36.5)$ & $42(32.6)$ & $47(39.2)$ & $49(38)$ & $42(36.5)$ \\
\hline & $\begin{array}{l}\text { Smoking } \\
\text { index, median }\end{array}$ & $\begin{array}{l}600 \\
(400-900)\end{array}$ & $\begin{array}{l}600 \\
(300-800)\end{array}$ & $\begin{array}{l}600 \\
(400-1000)\end{array}$ & $\begin{array}{l}600 \\
(400-1000)\end{array}$ & $\begin{array}{l}600 \\
(300-1000)\end{array}$ \\
\hline HR, median (IQR) & & 88(78-96.5) & $89(80-98.5)$ & $89(78-98)$ & $84(76-95)$ & $85(78-96)$ \\
\hline RR, median (IQR) & & $20(20-21)$ & $20(20-22)$ & $20(20-21.75)$ & $20(20-21)$ & $20(20-21)$ \\
\hline LTOT, n (\%) & & $246(49.9)$ & $75(58.1)$ & $65(54.2)$ & $55(42.6)$ & $5 I(44.3)$ \\
\hline \multirow[t]{3}{*}{ Daily treatment } & ICS, n (\%) & $176(35.7)$ & $52(40.3)$ & $42(35)$ & $38(29.5)$ & $44(38.3)$ \\
\hline & LABA, n (\%) & 176(35.7) & $52(40.3)$ & $42(35)$ & $38(29.5)$ & $44(38.3)$ \\
\hline & LAMA, n (\%) & $106(21.5)$ & $34(26.4)$ & $22(18.3)$ & $21(16.3)$ & $29(25.2)$ \\
\hline CAT, median (IQR) & & $18(14-26)$ & $20(15-27.5)$ & $18(14-26)$ & $17(13-25)$ & $19(14-26)$ \\
\hline mMRC, median (IQR) & & $2(I-3)$ & $2(I-3)$ & $2(I-3)$ & $2(I-3)$ & $2(I-3)$ \\
\hline $\begin{array}{l}\text { Moderate or severe exacerbation } \\
\text { history in previous year, median (IQR) }\end{array}$ & & $I(0-2)$ & $I(0-2)$ & $1.5(0.25-3)$ & $I(0-3)$ & $I(0-3)$ \\
\hline $\begin{array}{l}\text { Exacerbations leading to hospital or } \\
\text { emergency admission in previous year, } \\
\text { median (IQR) }\end{array}$ & & $I(0-2)$ & $I(0-2)$ & $I(0.25-3)$ & $I(0-2)$ & $I(0-2)$ \\
\hline \multirow[t]{4}{*}{$\mathrm{ABCD}$ assessment, $\mathrm{n}(\%)$} & A & $129(26.2)$ & $\mathrm{I}(0.8)$ & $40(31)$ & $2(1.6)$ & $86(66.7)$ \\
\hline & B & $120(24.3)$ & $\mathrm{I}(0.8)$ & $29(24.2)$ & $2(1.7)$ & $88(73.3)$ \\
\hline & C & $129(26.2)$ & $3(2.3)$ & $36(27.9)$ & $2(1.6)$ & $88(68.2)$ \\
\hline & $\mathrm{D}$ & $115(23.3)$ & $2(1.7)$ & $33(28.7)$ & $0(0)$ & $80(69.6)$ \\
\hline \multirow[t]{2}{*}{ Pattern of admission, n (\%) } & Outpatient service & $286(58)$ & $70(54.3)$ & $55(45.8)$ & $91(70.5)$ & $70(60.9)$ \\
\hline & Emergency & $207(42)$ & $59(45.7)$ & $65(54.2)$ & $38(29.5)$ & $45(39.1)$ \\
\hline
\end{tabular}

Notes: ${ }^{b}$ Patients were grouped by quartile absolute count $(0,0.05 \times 109 / \mathrm{L}, 0.17 \times 109 / \mathrm{L})$ of blood eosinophils and divided into group I, 2,3 and 4 (from low to high). $*$ Mean (SD, standard deviation).

Abbreviations: IQR, interquartile range: $25 \%-75 \%$; n, number; BMI, body mass index; ICS, inhaled corticosteroids; LAMA, long-acting muscarinic antagonist; LABA, longacting beta agonist; HR, heart rate; RR, respiratory rate; LTOT, long-term oxygen therapy; CAT, COPD assessment test; mMRC, modified British medical research council. 
Table 3 Patients' Laboratory Findings on Admission of Quartile-Percentage of Eosinophil Cohorts

\begin{tabular}{|c|c|c|c|c|c|c|c|}
\hline \multirow{2}{*}{$\begin{array}{l}\text { Variables } \\
\text { Groups }\end{array}$} & & \multicolumn{6}{|c|}{ Percentage of Peripheral Blood Eosinophil ${ }^{c}$} \\
\hline & & Overall & $\mathbf{I}$ & 2 & 3 & 4 & $P$ value \\
\hline \multirow{7}{*}{$\begin{array}{l}\text { Arterial } \\
\text { blood gas } \\
\text { analysis }\end{array}$} & $\mathrm{PH}$ & $\begin{array}{l}7.416 \\
(7.3835-7.447)\end{array}$ & $\begin{array}{l}7.411 \\
(7.370-7.449)\end{array}$ & $7.414(0.06)^{*}$ & $\begin{array}{l}7.416 \\
(7.388-7.444)\end{array}$ & $7.417(0.04 I)^{*}$ & 0.783 \\
\hline & $\mathrm{PaO} 2, \mathrm{mmHg}$ & $82(66.85-104)$ & $\begin{array}{l}87.05 \\
(66.85-103.75)\end{array}$ & $82.7(67-104)$ & $83(64.9-108.7)$ & $79.2(68-102)$ & 0.972 \\
\hline & $\mathrm{PaCO} 2, \mathrm{mmHg}$ & $41.2(36.5-50.55)$ & $\begin{array}{l}43.25 \\
(37.9-56.275)\end{array}$ & $41.2(34.8-53.7)$ & $40.8(36.8-46)$ & $40.9(36.4-47.1)$ & 0.122 \\
\hline & $\mathrm{SaO} 2 \%$ & $96.2(93-98.1)$ & $96(92.2-98)$ & $96.3(93.3-98.3)$ & $96(92-98)$ & $96.6(94-98.1)$ & 0.530 \\
\hline & $\mathrm{FiO} 2$ & $0.29(0.29-0.33)$ & $0.29(0.29-0.33)$ & $0.29(0.29-0.33)$ & $0.29(0.29-0.30)$ & $0.29(0.29-0.33)$ & 0.104 \\
\hline & $\mathrm{PaO} 2 / \mathrm{FiO} 2$ & $\begin{array}{l}286.207 \\
(234.483-347.414)\end{array}$ & $\begin{array}{l}286.078 \\
(237.972-338.054)\end{array}$ & $\begin{array}{l}290 \\
(231.034-351.515)\end{array}$ & $\begin{array}{l}295.238 \\
(243.8 I-359.31)\end{array}$ & $\begin{array}{l}278.276 \\
(227.586-344.828)\end{array}$ & 0.710 \\
\hline & $\mathrm{PaO} 2 / \mathrm{SaO} 2$ & $\begin{array}{l}0.854 \\
(0.712-1.058)\end{array}$ & $0.904(0.722-1.07)$ & $\begin{array}{l}0.856 \\
(0.713-1.054)\end{array}$ & $\begin{array}{l}0.856 \\
(0.7-1.102)\end{array}$ & $\begin{array}{l}0.821 \\
(0.723-7.04 I)\end{array}$ & 0.963 \\
\hline \multirow[t]{11}{*}{$\begin{array}{l}\text { Blood } \\
\text { routine }\end{array}$} & $\begin{array}{l}\text { Leukocyte } \\
\text { count, } 10^{9} / \mathrm{L}\end{array}$ & $7.31(5.54-10.125)$ & $\begin{array}{l}8.33 \\
(6.268-11.898)\end{array}$ & $8.59(6.47-\mid I .4 I)$ & $7.22(5.54-9.05)$ & $6.12(4.9-7.62)$ & 0.000 \\
\hline & $\begin{array}{l}\text { Neutrophil } \\
\text { count, } 10^{9} / \mathrm{L}\end{array}$ & $5.43(3.75-8.305)$ & $7.32(5.113-9.928)$ & $6.79(4.64-9.51)$ & $4.9(3.66-7.12)$ & $3.91(2.97-5.16)$ & 0.000 \\
\hline & $\begin{array}{l}\text { Lymphocyte } \\
\text { count, } 10^{9} / \mathrm{L}\end{array}$ & $1.06(0.7-1.5 \mathrm{I})$ & $0.7 I(0.46-1.153)$ & $1.03(0.7-1.49)$ & $1.19(0.88-1.6)$ & $1.18(0.89-1.76)$ & 0.000 \\
\hline & $\begin{array}{l}\text { Monocyte } \\
\text { count, } 10^{9} / \mathrm{L}\end{array}$ & $0.5(0.36-0.69)$ & $\begin{array}{l}0.43 \\
(0.2425-0.598)\end{array}$ & $0.55(0.38-0.76)$ & $0.54(0.43-0.67)$ & $0.48(0.37-0.66)$ & 0.000 \\
\hline & $\begin{array}{l}\text { Eosinophil } \\
\text { count, } 10^{9} / \mathrm{L}\end{array}$ & $0.05(0-0.17)$ & $0(0)$ & $0.02(0.01-0.03)$ & $0.1(0.08-0.14)$ & $0.26(0.2-0.4)$ & 0.000 \\
\hline & $\begin{array}{l}\text { Basophil } \\
\text { count, } 10^{9} / \mathrm{L}\end{array}$ & $0.02(0.01-0.03)$ & $0.01(0.01-0.02)$ & $0.01(0.01-0.03)$ & $0.02(0.01-0.04)$ & $0.03(0.02-0.04)$ & 0.000 \\
\hline & Neutrophil, \% & $76.1(66.4-83.9)$ & $\begin{array}{l}84.45 \\
(81.025-89.55)\end{array}$ & $79.3(74.1-85.1)$ & 7I.I(65.4-78.5) & 64.I(57.6-7I.4) & 0.000 \\
\hline & Lymphocyte, \% & $14.7(9.3-2 \mid .05)$ & $9.9(5.725-14.3)$ & $13(8.5-18.3)$ & $17.7(12-17.7)$ & $20.3(15.2-27.1)$ & 0.000 \\
\hline & Monocyte, \% & $6.9(5-8.8)$ & $4.8(3.1-6.45)$ & $6.5(4.9-8.5)$ & 7.6(5.9-9.5) & $0(6.5-8)$ & 0.000 \\
\hline & Eosinophil, \% & $0.7(0-2.55)$ & $0(0)$ & $0.3(0.1-0.4)$ & I.4(I-2) & $4.2(3.3-5.5)$ & 0.000 \\
\hline & Basophil, \% & $0.2(0.1-0.4)$ & $0.1(0.1-0.2)$ & $0.2(0.1-0.3)$ & $0.3(0.1-0.5)$ & $0.5(0.2-0.7)$ & 0.000 \\
\hline
\end{tabular}

Notes: 'Patients were grouped by quartile percentage $(0,0.7,2.55)$ of blood eosinophils and divided into group I, 2,3 and 4 (from low to high). *Mean (SD, standard deviation).

Abbreviations: IQR, interquartile range: 25\%-75\%; E\%, percent of blood eosinophil in white blood cell; E, absolute count of peripheral blood eosinophil.

in the quartile-percentage eosinophil cohorts (12 vs 10 days; $\mathrm{p}=0.005)$.

The secondary outcomes were the rate and duration of NIMV, comorbidities, mortality, and ICU admission. The frequencies of heart failure, respiratory failure, and pulmonary heart disease were found to be different between the two classified cohorts $(\mathrm{p}=0.004, \mathrm{p}=0.005 ; \mathrm{p}=0.002, \mathrm{p}=0.008$; $\mathrm{p}=0.005, \mathrm{p}=0.005$, respectively) (Tables 5 and 6 ). In the quartile-percentage eosinophil cohorts, patients in Group 1 experienced significantly higher rates of heart failure than those in Group 4 (48.4\% vs 28.5\%, p = 0.001), had higher frequencies of respiratory failure and pulmonary heart disease compared with those in Groups 3 and 4 in a pairwise comparison $(54.8 \%$ vs $34.8 \%, \mathrm{p}=0.002 ; 54.8 \%$ vs $35 \%, \mathrm{p}=$ 
Table 4 Patients' Laboratory Findings on Admission of Quartile-Count of Eosinophil Cohorts

\begin{tabular}{|c|c|c|c|c|c|c|c|}
\hline \multirow{2}{*}{$\begin{array}{l}\text { Variables } \\
\text { Groups }\end{array}$} & & \multirow[t]{2}{*}{ Overall } & \multicolumn{5}{|c|}{ Absolute Count of Peripheral Blood Eosinophil ${ }^{d}$} \\
\hline & & & $\mathbf{I}$ & 2 & 3 & 4 & $P$ value \\
\hline \multirow[t]{7}{*}{$\begin{array}{l}\text { Arterial blood } \\
\text { gas analysis }\end{array}$} & $\mathrm{PH}$ & $\begin{array}{l}7.416 \\
(7.3835-7.447)\end{array}$ & $7.4 I I(7.367-7.45)$ & $\begin{array}{l}7.421 \\
(7.387-7.456)\end{array}$ & $\begin{array}{l}7.412 \\
(7.385-7.439)\end{array}$ & $7.417(0.045)^{*}$ & 0.421 \\
\hline & $\mathrm{PaO} 2, \mathrm{mmHg}$ & $82(66.85-104)$ & $85(64.95-102)$ & $\begin{array}{l}88.5 \\
(67.925-107.3)\end{array}$ & $80(64.9-105)$ & $78(68-102)$ & 0.365 \\
\hline & $\begin{array}{l}\mathrm{PaCO} 2 \\
\mathrm{mmHg}\end{array}$ & $41.2(36.5-50.55)$ & $43.3(37.85-57)$ & $\begin{array}{l}40.3 \\
(34.75-51.175)\end{array}$ & $41.2(36.6-47.6)$ & $40.6(36.5-46.5)$ & 0.079 \\
\hline & $\mathrm{SaO} 2 \%$ & $96.2(93-98.1)$ & $96(92-98)$ & $97(94-98.65)$ & $96(92-98)$ & $96.6(94-98)$ & 0.125 \\
\hline & $\mathrm{FiO} 2$ & $0.29(0.29-0.33)$ & $0.29(0.29-0.33)$ & $0.29(0.29-0.33)$ & $0.29(0.29-0.315)$ & $0.29(0.29-0.33)$ & 0.286 \\
\hline & $\mathrm{PaO} 2 / \mathrm{FiO}_{2}$ & $\begin{array}{l}286.207 \\
(234.483-347.414)\end{array}$ & $\begin{array}{l}279.31 \\
(234.483-336.207)\end{array}$ & $\begin{array}{l}296.552 \\
(241.413-357.537)\end{array}$ & $\begin{array}{l}280.952 \\
(234.483-359.785)\end{array}$ & $\begin{array}{l}278.276 \\
(227.879-344.276)\end{array}$ & 0.338 \\
\hline & $\mathrm{PaO} 2 / \mathrm{SaO} 2$ & $\begin{array}{l}0.854 \\
(0.712-1.058)\end{array}$ & $0.88(0.707-1.046)$ & $0.904(0.72-1.097)$ & $\begin{array}{l}0.833 \\
(0.699-1.07 I)\end{array}$ & $\begin{array}{l}0.813 \\
(0.736-1.04 I)\end{array}$ & 0.455 \\
\hline \multirow[t]{11}{*}{ Blood routine } & $\begin{array}{l}\text { Leukocyte } \\
\text { count, } 10^{9} / \mathrm{L}\end{array}$ & $7.31(5.54-10.125)$ & $7.79(5.85-10.95)$ & $\begin{array}{l}8.49 \\
(5.913-11.398)\end{array}$ & $6.64(5.09-9.015)$ & $6.84(5.55-8.5)$ & 0.001 \\
\hline & $\begin{array}{l}\text { Neutrophil } \\
\text { count, } 10^{9} / \mathrm{L}\end{array}$ & $5.43(3.75-8.305)$ & $6.56(4.72-9.5 I)$ & $\begin{array}{l}6.71 \\
(4.2875-9.508)\end{array}$ & $4.63(3.475-6.92)$ & $4.43(3.35-6.09)$ & 0.000 \\
\hline & $\begin{array}{l}\text { Lymphocyte } \\
\text { count, } 10^{9} / \mathrm{L}\end{array}$ & I.06(0.7-I.5I) & $0.69(0.46-1.115)$ & $1.05(0.703-1.05)$ & $1.2 \mathrm{I}(0.87-1.665)$ & I.18(0.95-1.79) & 0.000 \\
\hline & $\begin{array}{l}\text { Monocyte } \\
\text { count, } 10^{9} / \mathrm{L}\end{array}$ & $0.5(0.36-0.69)$ & $0.4 \mathrm{I}(0.24-0.585)$ & $0.525(0.4-0.725)$ & $0.52(0.385-0.685)$ & $0.54(0.39-0.69)$ & 0.000 \\
\hline & $\begin{array}{l}\text { Eosinophil } \\
\text { count, } 10^{9} / \mathrm{L}\end{array}$ & $0.05(0-0.17)$ & $0(0)$ & $0.02(0.01-0.04)$ & $0.1(0.08-0.14)$ & $0.27(0.2 I-0.4 I)$ & 0.000 \\
\hline & $\begin{array}{l}\text { Basophil } \\
\text { count, } 10^{9} / \mathrm{L}\end{array}$ & $0.02(0.01-0.03)$ & $0.01(0.01-0.02)$ & $0.01(0.01-0.02)$ & $0.02(0.01-0.04)$ & $0.03(0.02-0.04)$ & 0.000 \\
\hline & Neutrophil, \% & $76.1(66.4-83.9)$ & $83.667(7.299)^{*}$ & $79.65(72.225-5.6)$ & $70.3(63.5-77.25)$ & $64.6(59.5-74.7)$ & 0.000 \\
\hline & Lymphocyte, \% & $14.7(9.3-2 \mid .05)$ & $10.4(6-14.6)$ & $12.45(8.5-17.475)$ & $18.3(12.3-26.95)$ & $19.7(13.1-26.2)$ & 0.000 \\
\hline & Monocyte, \% & $6.9(5-8.8)$ & $5.1(3.1-6.55)$ & $6.5(4.725-8.7)$ & 7.7(5.95-9.3) & $8.073(2.453)^{*}$ & 0.000 \\
\hline & Eosinophil, \% & $0.7(0-2.55)$ & $0(0)$ & $0.3(0.1-0.4)$ & $1.5(I-2.35)$ & $4.4(3.3-5.6)$ & 0.000 \\
\hline & Basophil, \% & $0.2(0.1-0.4)$ & $0.1(0.1-0.2)$ & $0.2(0.1-0.2)$ & $0.3(0.2-0.6)$ & $0.4(0.2-0.6)$ & 0.000 \\
\hline
\end{tabular}

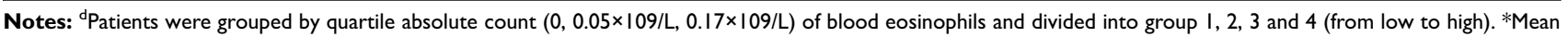
(SD, standard deviation).

Abbreviations: IQR, interquartile range: $25 \%-75 \%$; E\%, percent of blood eosinophil in white blood cell; E, absolute count of peripheral blood eosinophil.

$0.003 ; 50.8 \%$ vs $32.2 \%, \mathrm{p}=0.004 ; 50.8 \%$ vs $34.1 \%, \mathrm{p}=$ 0.008). In the eosinophil count cohorts, patients in Group 1 had significantly higher rates of heart failure, respiratory failure, and pulmonary heart disease than those in Groups 3 and 4 in a pairwise comparison $(48.1 \%$ vs $30.2 \%, p=0.003$; $48.1 \%$ vs $30.4 \%, \mathrm{p}=0.005 ; 55.8 \%$ vs $37.2 \%, \mathrm{p}=0.003$; $55.8 \%$ vs $37.4 \%, \mathrm{p}=0.004 ; 51.9 \%$ vs $34.1 \%, \mathrm{p}=0.004$ $51.9 \%$ vs $33 \%, p=0.003)$.
Except for the duration of NIMV, the proportion of ICU admission, NIMV, and mortality were found to vary among groups $(\mathrm{p}=0.009, \mathrm{p}=0.01 ; \mathrm{p}=0.011, \mathrm{p}=0.005$; $\mathrm{p}=0.05, \mathrm{p}=0.283$ ) (Tables 5 and 6). There was a significantly higher rate of NIMV in Group 1 than in Group 4 in the percentage of eosinophil cohorts $(29.5 \%$ vs $23.6 \%, \mathrm{p}=0.007)$. Comparable results were found in Group 1 compared to Group 3 in the eosinophil count 
Table 5 Comparison of Clinical Outcomes of Quartile-Percentage of Eosinophil Cohorts

\begin{tabular}{|c|c|c|c|c|c|c|c|}
\hline \multirow{2}{*}{$\begin{array}{l}\text { Variables } \\
\text { Groups }\end{array}$} & & \multirow[t]{2}{*}{ Sum } & \multicolumn{4}{|c|}{ Percentage of Peripheral Blood Eosinophil } & \multirow[t]{2}{*}{$P$ value } \\
\hline & & & $\mathbf{I}$ & 2 & 3 & 4 & \\
\hline Participants, $\mathrm{n}$ & & 493 & 124 & $|3|$ & 115 & 123 & 493 \\
\hline $\begin{array}{l}\text { Stay of hospital, median } \\
\text { (IQR) }\end{array}$ & & II (9-14) & $12(9.25-14)$ & II (8-14) & II (9-I4) & $10(9-13) *$ & 0.01 \\
\hline ICU admission, n (\%) & & $6(1.2)$ & $5(1)$ & $0(0)$ & $0(0)$ & $\mathrm{I}(0.2)$ & $0.009 *$ \\
\hline Mortality, n (\%) & & $5(1)$ & $0(0)$ & $4(0.8)$ & $\mathrm{I}(0.2)$ & $0(0)$ & $0.05^{*}$ \\
\hline \multirow[t]{10}{*}{ Comorbidity, n (\%) } & $\begin{array}{l}\text { Cardiovascular } \\
\text { disease }\end{array}$ & $37 I(75.3)$ & $101(81.5)$ & $99(75.6)$ & $85(73.9)$ & $86(69.9)$ & 0.207 \\
\hline & $\begin{array}{l}\text { Ischemic heart } \\
\text { disease }\end{array}$ & $73(14.8)$ & $15(12.1)$ & $21(16)$ & $19(16.5)$ & $18(14.6)$ & 0.775 \\
\hline & Heart failure & $188(38.1)$ & $60(48.4)$ & $56(42.7)$ & $37(32.2)$ & $35(28.5)$ & 0.004 \\
\hline & Hypertension & $207(42)$ & $5 I(4 I . I)$ & $56(42.7)$ & $53(46.1)$ & $47(38.2)$ & 0.67 \\
\hline & $\begin{array}{l}\text { Peripheral vascular } \\
\text { disease }\end{array}$ & $63(12.8)$ & $18(\mid 4.5)$ & $13(9.9)$ & $15(13)$ & $17(13.8)$ & 0.714 \\
\hline & Arrhythmia & $78(15.8)$ & $25(20.2)$ & $16(12.2)$ & $19(16.5)$ & $18(14.6)$ & 0.365 \\
\hline & Diabetes & $8 I(16.4)$ & $19(15.3)$ & $28(21.4)$ & $19(16.5)$ & $15(12.2)$ & 0.258 \\
\hline & Bronchiectasis & $87(17.6)$ & $21(16.9)$ & $27(20.6)$ & $19(16.5)$ & $20(17.3)$ & 0.776 \\
\hline & Respiratory failure & $215(43.6)$ & $68(54.8)$ & $64(48.9)$ & $40(34.8)$ & $43(35)$ & 0.002 \\
\hline & $\begin{array}{l}\text { Pulmonary heart } \\
\text { disease }\end{array}$ & 203(4I.2) & $63(50.8)$ & $6 I(46.6)$ & $37(32.2)$ & $42(34.1)$ & 0.005 \\
\hline \multirow{3}{*}{$\begin{array}{l}\text { Mechanical ventilation, } \\
\text { n (\%) }\end{array}$} & NIMV & $147(29.8)$ & $49(29.5)$ & $43(32.8)$ & $26(22.6)$ & $29(23.6)$ & 0.011 \\
\hline & IMV & $4(0.8)$ & $2(0.4)$ & $\mathrm{I}(0.2)$ & $0(0)$ & $\mathrm{I}(0.2)$ & $0.757^{*}$ \\
\hline & Duration of NIMV & $\begin{array}{l}238 \\
(139.75-309.75)\end{array}$ & $\begin{array}{l}235 \\
(124.5-305.5)\end{array}$ & $\begin{array}{l}274.47 \\
(139.482) * *\end{array}$ & $\begin{array}{l}282.5 \\
(193.5-339)\end{array}$ & $\begin{array}{l}214.29 \\
(103.24 I)\end{array}$ & 0.381 \\
\hline
\end{tabular}

Notes: ${ }^{e}$ Patients were grouped by quartile percentage $(0,0.7,2.55)$ of blood eosinophils and divided into group I, 2,3 and 4 (from low to high). *Fisher's exact probability method; **Mean (SD, standard deviation).

Abbreviations: IQR, interquartile range: $25 \%-75 \%$; N, number; E\%, percent of blood eosinophil in white blood cell; E, absolute count of peripheral blood eosinophil; ICU, intensive care unit; NIMV, noninvasive mechanical ventilation; IMV, invasive mechanical ventilation.

cohorts $(41.1 \%$ vs $21.7 \%, \mathrm{p}=0.001)$. No difference was found in mortality or ICU admission in a pairwise comparison $(\mathrm{p}>0.008)$.

Kaplan-Meier analyses identified a significant difference between eosinophil groups in length of hospital stay in both quartile-percentage and absolute count of eosinophil groups $(\mathrm{P}<0.023 ; \mathrm{P}<0.035)$ (Figure $\mathrm{S} 1$ and $\underline{\mathrm{S} 2}$, Tables S1-S4). The median hospital stays were both 11 days for the absolute count and quartile-percentage eosinophil groups. Using the median hospital stay (11 days) as the cutoff value, ROC analysis of the cutoff values of blood eosinophil for longer hospital stay at $\geq 11$ days were as follows: percentage of eosinophil $<0.45$ was associated with a longer hospital stay (AUC: 0.585 , sensitivity: 0.534, specificity: $0.613, \mathrm{P}=$ 0.001 ), while an eosinophil count of $<0.025 \times 10^{9} / \mathrm{L}$ (AUC: 0.579 , sensitivity: 0.336, specificity: 0.805, $\mathrm{P}=$ 0.003) was associated with a longer hospital stay. Details are shown in Figure S3 and Table S5.

A sensitivity analysis was performed to address potential bias from analytical methods. Associations between eosinophil classification and clinical outcomes were further surveyed using different cutoffs to define eosinophilia. 
Table 6 Comparison of Clinical Outcomes of Quartile-Count of Eosinophil Cohorts

\begin{tabular}{|c|c|c|c|c|c|c|c|}
\hline \multirow{2}{*}{$\begin{array}{l}\text { Variables } \\
\text { Groups }\end{array}$} & & \multirow[t]{2}{*}{ Overall } & \multicolumn{4}{|c|}{ Absolute Count of Peripheral Blood Eosinophil ${ }^{f}$} & \multirow[b]{2}{*}{$P$ value } \\
\hline & & & $\mathbf{I}$ & 2 & 3 & 4 & \\
\hline Participants, $n$ & & 493 & 129 & 120 & 129 & 115 & \\
\hline $\begin{array}{l}\text { Stay of hospital, median } \\
\text { (IQR) }\end{array}$ & & $11(9-14)$ & $12(10-14)$ & $10(8-14)$ & $\mathrm{II}(8-13)$ & $10(9-13)$ & 0.002 \\
\hline ICU admission, n (\%) & & $6(1.2)$ & $5(1)$ & $0(0)$ & $0(0)$ & $\mathrm{I}(0.2)$ & 0.01 \\
\hline Mortality, n (\%) & & $5(1)$ & $\mathrm{I}(0.2)$ & $3(0.6)$ & $\mathrm{I}(0.2)$ & $0(0)$ & $0.283^{*}$ \\
\hline \multirow[t]{10}{*}{ Comorbidity, n (\%) } & $\begin{array}{l}\text { Cardiovascular } \\
\text { disease }\end{array}$ & $37 I(75.3)$ & $103(79.8)$ & $91(75.8)$ & $97(75.2)$ & $80(69.6)$ & 0.325 \\
\hline & $\begin{array}{l}\text { Ischemic heart } \\
\text { disease }\end{array}$ & $73(14.8)$ & $15(\mid 1.6)$ & $20(16.7)$ & $19(14.7)$ & $19(16.5)$ & 0.663 \\
\hline & Heart failure & $188(38.1)$ & $62(48.1)$ & $52(43.3)$ & $39(30.2)$ & $35(30.4)$ & 0.005 \\
\hline & Hypertension & 207(42) & $50(38.8)$ & $52(43.3)$ & $57(44.2)$ & $48(4 I .7)$ & 0.824 \\
\hline & $\begin{array}{l}\text { Peripheral vascular } \\
\text { disease }\end{array}$ & $63(12.8)$ & $18(14)$ & II (9.2) & $18(14)$ & $16(13.9)$ & 0.613 \\
\hline & Arrhythmia & $78(15.8)$ & $24(18.6)$ & $18(15)$ & $20(15.5)$ & $16(13.9)$ & 0.767 \\
\hline & Diabetes & $81(16.4)$ & $20(\mid 5.5)$ & $25(20.8)$ & $21(16.3)$ & $15(13)$ & 0.433 \\
\hline & Bronchiectasis & $87(17.6)$ & $22(17.1)$ & $24(20)$ & $21(16.3)$ & $20(17.4)$ & 0.884 \\
\hline & Respiratory failure & $215(43.6)$ & $72(55.8)$ & $52(43.3)$ & $48(37.2)$ & $43(37.4)$ & 0.008 \\
\hline & $\begin{array}{l}\text { Pulmonary heart } \\
\text { disease }\end{array}$ & $203(4 I .2)$ & $67(51.9)$ & $54(45)$ & $44(34.1)$ & $38(33)$ & 0.005 \\
\hline \multirow{3}{*}{$\begin{array}{l}\text { Mechanical ventilation, } \\
\text { n (\%) }\end{array}$} & NIMV & 147(29.8) & $53(4 I . I)$ & $37(30.8)$ & $28(2 \mid .7)$ & $29(25.2)$ & 0.005 \\
\hline & IMV & $4(0.8)$ & $2(0.4)$ & $\mathrm{I}(0.2)$ & $0(0)$ & $\mathrm{I}(0.2)$ & $0.657^{*}$ \\
\hline & Duration of NIMV & $\begin{array}{l}238 \\
(139.75-309.75)\end{array}$ & $\begin{array}{l}237 \\
(138.5-320)\end{array}$ & $\begin{array}{l}242.49 \\
(148.295)^{* *}\end{array}$ & $\begin{array}{l}242.7 I \\
(116.665)^{* *}\end{array}$ & $\begin{array}{l}241 \\
(164.25-304.25)\end{array}$ & 0.994 \\
\hline
\end{tabular}

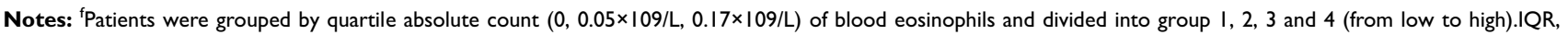
interquartile range: $25 \%-75 \%$; *Fisher's exact probability method; **Mean (SD, standard deviation).

Abbreviations: N, number; E\%, percent of blood eosinophil in white blood cell; E, absolute count of peripheral blood eosinophil; ICU, intensive care unit; NIMV, noninvasive mechanical ventilation; IMV, invasive mechanical ventilation.

Alternative cutoffs of $2 \%, 100 / \mu \mathrm{L}$, and $300 / \mu \mathrm{L}$ were used to verify the difference in the hospital stay length, ICU admission, rate, and duration of noninvasive ventilation, comorbidities, and mortality. Analysis of the $2 \%$ cutoff showed there are 151 patients $(30.63 \%)$ with eosinophil $\geq 2 \%$. Patients with eosinophil $<2 \%$ experience longer hospital stays and more respiratory failures (Table S6). There are 177 patients (35.9\%) with eosinophil $\geq 100 / \mu \mathrm{L}$. Patients with eosinophil counts $<100 / \mu \mathrm{L}$ were associated with longer hospital stays and higher proportions of NIMV, respiratory failure, heart failure, and pulmonary heart disease (Table S7). Only 43 patients $(8.7 \%)$ had eosinophil $\geq 300 / \mu L$. Patients with eosinophil counts $<300 / \mu \mathrm{L}$ were associated with longer durations of NIMV, and higher proportions of heart failure and pulmonary heart disease (Table S8). Different ways of grouping showed comparable results; patients with lower eosinophil experience poorer clinical outcomes in patients with AECOPD.

\section{Discussion}

We analyzed the clinical characteristics and outcomes of AECOPD patients according to the percent and absolute 
count of eosinophils based on routine blood counts. We found that patients with higher eosinophil levels experienced better clinical outcomes. A significantly higher proportion of COPD patients with lower eosinophil counts required a longer hospital stay, NIMV, and experienced more complications. Patients with lower eosinophilic COPD exhibited a higher rate of heart failure, respiratory failure, and pulmonary heart disease than those in the higher eosinophilic COPD group.

There are several explanations for why COPD patients with lower eosinophil counts experienced poorer outcomes. First, lower eosinophilic COPD patients had higher neutrophil counts in our analysis. Neutrophilia is known to be a marker of bacterial infection, which is a common cause of exacerbation. COPD exacerbations caused by bacterial infection are also associated with longer hospital stays. $^{4,24,27}$ Collinearity diagnosis was performed on the logistic regression model to verify the correlation between eosinophil and neutrophil. There was no collinearity between the eosinophil count, neutrophil count, percentage of eosinophil, and neutrophil variation (Table S9). Therefore, we believe that neutrophil and eosinophil are independent factors. Second, an appropriate treatment with antibiotics and systemic corticosteroids can shorten recovery time and hospital stay. The decision for antibiotics and systemic corticosteroids used in our study was based on white blood cell counts, neutrophil levels, inflammatory biomarkers, patient signs and symptoms, chest imaging, and general clinical practice, without controlled peripheral eosinophils. Most patients in our study were prescribed antibiotics. However, lower eosinophilic COPD patients had a significantly higher frequency of systemic corticosteroid treatment. It has been reported that exacerbations associated with an increase in sputum or blood eosinophil levels may be more responsive to systemic steroids. ${ }^{28}$

Additionally, two recent studies reported that glucocorticoids might be less effective in AECOPD patients with lower levels of blood eosinophils. ${ }^{4,29}$ In this study, higher eosinophilic COPD patients may have benefitted from receiving more systemic steroids. More prospective trials are needed to verify this assertion.

Although the proportion of ICU admissions and mortality were different among the groups, no difference was found in the pairwise comparison. The number of ICU admissions and mortalities in our study was only 6 and 5 , respectively. Moreover, there were no participants in some groups. As such, the validity and reliability of these two analyses are limited. Thus, a study with larger sample size is needed.

In an analysis of the SubPopulations and InteRmediate Outcome Measures In COPD Study (SPIROMICS), significant differences were found in age, sex, BMI, percentage, predicted forced expiratory volume in one second $\left(\mathrm{ppFEV}_{1}\right), \mathrm{FEV}_{1} / \mathrm{FVC}$ ratio and smoking. However, there is no evidence of a GOLD stage between lower eosinophil $(<200 / \mu \mathrm{L})$ and higher eosinophil $(\geq 200 / \mu \mathrm{L})$ groups. $^{14}$ SPIROMICS was a retrospective observational cohort study that enrolled patients with a smoking history of at least 20 packs of cigarettes per year. Patients exhibited symptoms, exacerbations, activity limitations, and radiological evidence of airway disease. However, preserved lung functions not meeting the criteria for COPD diagnosis were included. These early COPD participants may have influenced the results. In a retrospective, observational study conducted in the ICU, patients with noneosinophilic COPD had a higher rate of NIMV on admission, NIMV failure, ICU mortality, arrhythmia, and a longer ICU stay than those with eosinophilic COPD. ${ }^{18}$ In this study, COPD patients were classified according to eosinophil levels (eosinophilic $>2 \%$ or non-eosinophilic $\leq$ $2 \%$ ). However, some patients were treated with antibiotics or steroids before ICU admission, which may have affected the results. Singh et $\mathrm{al}^{5}$ reported on COPD subjects with eosinophils $\geq 2 \%$, who were characterized by older age, a higher proportion of males, higher $\mathrm{ppFEV}_{1}$, fewer current smokers, better scores on the St. George's Respiratory Questionnaire (SGRQ) and mMRC than noneosinophil COPD and healthy control groups. Data from this analysis were from the Evaluation of COPD Longitudinally to Identify Predictive Surrogate Endpoints (ECLIPSE) study, which enrolled GOLD stage II-IV COPD patients with a smoking history $\geq 10$ pack of cigarettes per year. The ECLIPSE study was a 3-year investigation, involving subjects $>75$ years old with severe complications, who might not complete the study and were excluded. Data from those with mild COPD, older age, and those with severe complications were absent in this study.

However, Couillard et $\mathrm{al}^{30}$ reported that there was no significant difference in sex, age, smoking, home oxygen use, comorbidity, lung function, GOLD stage, and hospitalization for COPD in the previous year between the two COPD phenotypes. Moreover, there was no difference in length of hospital stay. That was a retrospective observational study that enrolled patients who were hospitalized 
for AECOPD. Eosinophils $\geq 200 / \mu \mathrm{L}$ or $\geq 2 \%$ was considered as the cutoff for group allocation. Participants with a history of asthma and bronchiectasis, admission for pneumonia, therapy for systemic corticosteroids between $1 \mathrm{hr}$ and $48 \mathrm{hrs}$ before admission were excluded. However, the use of antibiotics was not detailed. Although the use of antibiotics would not directly affect the absolute eosinophil count, it would influence the eosinophil percentage of total white blood cells.

Recently, Ko et al found that an eosinophil value of $<$ $0.144 \times 109 / \mathrm{L}$ or $<2 \%$ on admission was associated with longer hospital stays for AECOPD independent of age, lung function and previous hospital admissions. ${ }^{22}$ The median of the absolute eosinophil count, percent eosinophil, and hospital stay were $0.11 \times 10^{9} / \mathrm{L}, 1 \%$ and 5 days, respectively. That was a single-center study, and not all subjects had eosinophil count data, but the results were similar to our research. MacDonald et $\mathrm{al}^{21}$ found no significant difference in baseline characteristics between patients with low $(<50 / \mathrm{mL})$, normal $(50-150 / \mathrm{mL})$, or high $(>150 / \mathrm{mL})$ blood eosinophils in two cohorts. Patients with low eosinophil counts were associated with infection $(91 \%$ vs $51.9 \%, \mathrm{P}<0.001)$, longer hospital stay (7 vs 4 days, $\mathrm{P}<0.001$ ), and lower 12-month survival $(82.4 \%$ vs $90.7 \%, \mathrm{P}<0.028)$ than those in high eosinophil counts group.

Our study had some limitations. First, for low eosinophil levels in our study, we did not group patients according to $2 \%$ blood eosinophils as in previous investigations. Grouping according to the $2 \%$ cutoff could have led to several differences in the number of participants and induced an imbalance in the results. However, we found comparable results in a sensitivity analysis using the $2 \%$ cutoff. Second, the use of steroids was not according to eosinophil levels but determined by the physician according to patient signs and symptoms. The rate of steroid use was different among the groups. Third, the diagnosis of COPD was based on medical history records of spirometry; patients with asthma were excluded. Spirometry results were not recorded on admission because some patients were not able to take the test. Fourth, most patients enrolled in our study had poor symptom scores and were in stage B or D of the refined ABCD assessment. As such, our results cannot be applied to all stages of COPD. Finally, this was a prospective observational study of patients with AECOPD, and we used the data to assess the effect of peripheral blood eosinophil on their hospital stays during acute admissions.
Our study also had several advantages. First, it was a prospective multicenter study, with a large sample of AECOPD patients recruited from three teaching hospitals. Second, our research excluded patients with histories of steroid use. Corticosteroids affect eosinophil levels and induce eosinopenia. Thus, we excluded patients who possibly had taken steroids before enrollment. Third, although several comparative studies investigating eosinophilic COPD have been published, all have been retrospective analyses ${ }^{18,20,31,32}$ and included patients taking steroids before enrollment. Fourth, we did sensitivity analysis using alternativity cutoffs at $2 \%, 100 / \mu \mathrm{L}$, and $300 / \mu \mathrm{L}$ to avoid bias. Finally, our results suggest that lower peripheral eosinophil levels are associated with poor clinical outcomes. This information will aid clinicians who must evaluate and predict the clinical course of patients hospitalized for AECOPD.

\section{Conclusion}

Identifying biomarkers of AECOPD could be useful in classifying exacerbation phenotypes. Lower-eosinophilic COPD inpatients can be more severely ill, experience longer hospital stays, a higher rate of NIMV, and more complications. A lower eosinophilic state can be a helpful indicator to predict outcomes of COPD and may be useful for the management of patients who experience AECOPD. More studies are needed to evaluate if peripheral blood eosinophil can guide the use of antibiotics and corticosteroids.

\section{Abbreviations}

COPD, chronic obstructive pulmonary disease; BMI, body-mass index; $\mathrm{ppFEV}_{1}$, percent of predicted forced expiratory volume in 1s; GOLD, Global Initiative for Chronic Obstructive Lung Disease.

\section{Data Sharing Statement}

The datasets used and/or analyzed during the current study are available from the corresponding author on reasonable request.

\section{Acknowledgements}

This study was partially funded by the Science and Technology Support Project of Sichuan Province (2015SZ0234-3). We thank all the people who participated in this study. 


\section{Author Contributions}

All authors contributed to data analysis, drafting or revising the article, gave final approval of the version to be published, and agree to be accountable for all aspects of the work. H-XW and D-YC initiated and coordinated the study. K-QZ were responsible for the data collection and data analysis. Studies were reviewed by D-YC. H-XW wrote the first draft of the manuscript. All the authors were involved in the interpretation of the analyses and gave input to the final manuscript.

\section{Funding}

This study was partially funded by the Science and Technology Support Project of Sichuan Province (2015SZ0234-3).

\section{Disclosure}

The authors declare that they have no conflicts of interest regarding this manuscript.

\section{References}

1. The Global Initiative for Chronic Obstructive Lung Disease (GOLD). Global strategy for diagnosis, management and prevention of COPD 2019 UPDATE+. https://goldcopd.org/wp-content/uploads/2018/11/ GOLD-2019-v1.7-FINAL-14Nov2018-WMS.pdf. Accessed December 19, 2019.

2. Wedzicha JA, Seemungal TA. COPD exacerbations: defining their cause and prevention. Lancet. 2007;370(9589):786-796. doi:10.1016/ S0140-6736(07)61382-8

3. Seemungal TA, Donaldson GC, Paul EA, Bestall JC, Jeffries DJ, Wedzicha JA. Effect of exacerbation on quality of life in patients with chronic obstructive pulmonary disease. Am J Respir Crit Care Med. 1998;157(5 Pt 1):1418-1422. doi:10.1164/ajrccm.157.5.9709032

4. Bafadhel M, McKenna S, Terry S, et al. Acute exacerbations of chronic obstructive pulmonary disease: identification of biologic clusters and their biomarkers. Am J Respir Crit Care Med. 2011;184 (6):662-671. doi:10.1164/rccm.201104-0597OC

5. Singh D, Kolsum U, Brightling CE, et al. Eosinophilic inflammation in COPD: prevalence and clinical characteristics. Eur Respir J. 2014;44:1697-1700. doi:10.1183/09031936.00162414

6. Brightling CE, McKenna S, Hargadon B, et al. Sputum eosinophilia and the short term response to inhaled mometasone in chronic obstructive pulmonary disease. Thorax. 2005;60:193-198. doi:10.1136/ thx.2004.032516

7. Brightling CE, Monteiro W, Ward R, et al. Sputum eosinophilia and short-term response to prednisolone in chronic obstructive pulmonary disease: a randomised controlled trial. Lancet. 2000;356:1480-1485. doi:10.1016/S0140-6736(00)02872-5

8. Leigh R, Pizzichini MM, Morris MM, Maltais F, Hargreave FE, Pizzichini E. Stable COPD: predicting benefit from high-dose inhaled corticosteroid treatment. Eur Respir J. 2006;27:964-971. doi:10.1183/ 09031936.06.00072105

9. Pizzichini E, Pizzichini MM, Gibson P, et al. Sputum eosinophilia predicts benefit from prednisone in smokers with chronic obstructive bronchitis. Am J Respir Crit Care Med. 1998;158:1511-1517. doi:10.1164/ajrccm.158.5.9804028
10. Pascoe S, Locantore N, Dransfield MT, Barnes NC, Pavord ID. Blood eosinophil counts, exacerbations, and response to the addition of inhaled fluticasone furoate to vilanterol in patients with chronic obstructive pulmonary disease: a secondary analysis of data from two parallel randomised controlled trials. Lancet Respir Med. 2015;3:435-442. doi:10.1016/S2213-2600(15)00106-X

11. Pavord ID, Lettis S, Locantore N, et al. Blood eosinophils and inhaled corticosteroid/long-acting $\beta-2$ agonist efficacy in COPD. Thorax. 2016;71:118-125. doi:10.1136/thoraxjnl-2015-207021

12. Watz H, Tetzla K, Wouters EF, et al. Blood eosinophil count and exacerbations in severe chronic obstructive pulmonary disease after withdrawal of inhaled corticosteroids: a post-hoc analysis of the WISDOM trial. Lancet Respir Med. 2016;4:390-398. doi:10.1016/ S2213-2600(16)00100-4

13. Barnes NC, Sharma R, Lettis S, Calverley PM. Blood eosinophils as a marker of response to inhaled corticosteroids in COPD. Eur Respir J. 2016;47:1374-1382. doi:10.1183/13993003.01370-2015

14. Hastie AT, Martinez FJ, Curtis JL, et al. Association of sputum and blood eosinophil concentrations with clinical measures of COPD severity: an analysis of the SPIROMICS cohort. Lancet Respir Med. 2017;5(12):956-967. doi:10.1016/S2213-2600(17)30432-0

15. Regan EA, Hokanson JE, Murphy JR, et al. Genetic epidemiology of COPD (COPDGene) study design. COPD J Chronic Obstr Pulm Dis. 2011;7:32-43. doi:10.3109/15412550903499522

16. Vestbo J, Anderson W, Coxson HO, et al. Evaluation of COPD longitudinally to identify predictive surrogate end-points (ECLIPSE). Eur Respir J. 2008;31:869-873. doi:10.1183/09031936.00111707

17. Bafadhel M, Peterson S, De Blas MA, et al. Predictors of exacerbation risk and response to budesonide in patients with chronic obstructive pulmonary disease: a post-hoc analysis of three randomised trials. Lancet Respir Med. 2018;6(2):117-126. doi:10.1016/S2213-2600(18)30006-7

18. Saltürk C, Karakurt Z, Adiguzel N, et al. Does eosinophilic COPD exacerbation have a better patient outcome than non-eosinophilic in the intensive care unit? Int $J$ Chron Obstruct Pulmon Dis. 2015;10:1837-1846. doi:10.2147/COPD.S88058

19. Kang HS, Rhee CK, Kim SK, et al. Comparison of the clinical characteristics and treatment outcomes of patients requiring hospital admission to treat eosinophilic and neutrophilic exacerbations of COPD. Int J Chron Obstruct Pulmon Dis. 2016;11:2467-2473. doi:10.2147/COPD.S116072

20. Duman D, Aksoy E, Agca MC, et al. The utility of inflammatory markers to predict readmissions and mortality in COPD cases with or without eosinophilia. Int $J$ Chron Obstruct Pulmon Dis. 2015;10:2469-2478. doi:10.2147/COPD.S90330

21. MacDonald MI, Osadnik CR, Bulfin L, et al. Low and high blood eosinophil counts as biomarkers in hospitalized acute exacerbations of COPD. CHEST. 2019;156(1):92-100. doi:10.1016/j.chest.2019.02.406

22. Ko FWS, Chan KP, Ngai J, et al. Blood eosinophil count as a predictor of hospital length of stay in COPD exacerbations. Respirology. 2019. doi:10.1111/resp. 13660

23. Rahimi-Rad MH, Asgari B, Hosseinzadeh N, Eishi A. Eosinopenia as a marker of outcome in acute exacerbations of chronic obstructive pulmonary disease. Maedica (Buchar). 2015;10(1):10-13.

24. Holland M, Alkhalil M, Chandromouli S, Janjua A, Babores M. Eosinopenia as a marker of mortality and length of stay in patients admitted with exacerbations of chronic obstructive pulmonary disease. Respirology. 2010;15(1):165-167. doi:10.1111/resp.2010.15.issue-1

25. Hasegawa K, Camargo CA Jr. Prevalence of blood eosinophilia in hospitalized patients with acute exacerbation of COPD. Respirology. 2016;21(4):761-764. doi:10.1111/resp.12724

26. Price D, Rigazio A, Postma D, et al. Blood eosinophilia and the number of exacerbations in COPD patients [abstract]. Eur Respir $J$. 2014;44(Suppl. 58):4416. doi:10.1183/09031936.00003814 
27. Chang C, Zhu H, Shen N, et al. Bacterial infection, airway and systemic inflammation and clinical outcomes before and after treatment of AECOPD, a longitudinal and cross-sectional study. COPD. 2015;12(1):19-30. doi:10.3109/15412555.2014.898043

28. Bafadhel M, McKenna S, Terry S, et al. Blood eosinophils to direct corticosteroid treatment of exacerbations of chronic obstructive pulmonary disease: a randomized placebo controlled trial. $\mathrm{Am}$ $J$ Respir Crit Care Med. 2012;186:48-55. doi:10.1164/rccm. 201108-1553OC

29. Hurst JR, Vestbo J, Anzueto A, et al. Susceptibility to exacerbation in chronic obstructive pulmonary disease. N Engl J Med. 2010;363 (12):1128-1138. doi:10.1056/NEJMoa0909883
30. Couillard S, Larivée P, Courteau J, Vanasse A. Eosinophils in COPD exacerbations are associated with increased readmissions. Chest. 2017;151(2):366-373. doi:10.1016/j.chest.2016.10.003

31. Bafadhel M, Greening NJ, Harvey-Dunstan TC, et al. Blood eosinophils and outcomes in severe hospitalized exacerbations of COPD. Chest. 2016;150(2):320-328. doi:10.1016/j.chest.2016.01.026

32. Serafino-Agrusa L, Scichilone N, Spatafora M, Battaglia S. Blood eosinophils and treatment response in hospitalized exacerbations of chronic obstructive pulmonary disease: a case-control study. Pulm Pharmacol Ther. 2016;37:89-94. doi:10.1016/j.pupt.2016.03.004

\section{Publish your work in this journal}

The International Journal of COPD is an international, peer-reviewed journal of therapeutics and pharmacology focusing on concise rapid reporting of clinical studies and reviews in COPD. Special focus is given to the pathophysiological processes underlying the disease, intervention programs, patient focused education, and self management protocols. This journal is indexed on PubMed Central, MedLine and CAS. The manuscript management system is completely online and includes a very quick and fair peer-review system, which is all easy to use. Visit http://www.dovepress.com/testimonials.php to read real quotes from published authors. 